\title{
Total Curvatures of Model Surfaces Control Topology of Complete Open Manifolds with Radial Curvature Bounded Below. I*广
}

\author{
Kei KONDO · Minoru TANAKA \\ Dedicated to Professor Karsten Grove on the occasion of his sixtieth birthday.
}

\begin{abstract}
We investigate the finiteness structure of a complete open Riemannian $n$-manifold $M$ whose radial curvature at a base point of $M$ is bounded from below by that of a non-compact von Mangoldt surface of revolution with its total curvature greater than $\pi$. We show, as our main theorem, that all Busemann functions on $M$ are exhaustions, and that there exists a compact subset of $M$ such that the compact set contains all critical points for any Busemann function on $M$. As corollaries by the main theorem, $M$ has finite topological type, and the isometry group of $M$ is compact.
\end{abstract}

\section{Introduction}

We have a great interest in the relationship between radial (sectional) curvature geometry and pure sectional curvature geometry. The difficulty in geometry of radial curvatures from a given point is that one has to look around such manifolds only at the base point. For example, on the Alexandrov - Toponogov comparison theorem, abbreviated to GTCTII (see Theorem 2.2 in Section 2), in such a comparison geometry, all geodesic triangles must have the base point as one of vertices. Thus, radial curvature geometry does not have, in a sense, homogeneity on curvature like pure sectional curvature geometry, which has all curvatures everywhere bounded below by some constant so that the AlexandrovToponogov comparison theorem in such a pure geometry holds for all geodesic triangles. Despite such a difficulty in radial curvature geometry, we have seen the new worlds (see GW, Gap Theorem], and also Katz and the first author' [KK]). In particular, after the work [IMS], and also Sinclair and the second author' [SiT], the difficulty in radial curvature geometry increases more, for more general class of models, von Mangoldt surfaces of revolution (defined below), and 2-spheres of revolution are employed as reference spaces

\footnotetext{
*Mathematics Subject Classification (2000): 53C20, 53C21.

$\dagger$ 'Keywords: Alexandrov - Toponogov comparison theorem, Busemann function, Cohn-Vossen, cut locus, exhaustion of functions, finite topological type, isometry group, Lipschitz function, mass of rays, radial curvature, Riemannian manifold, total curvature of surface, von Mangoldt surface of revolution
} 
in comparison theorems of radial curvature geometry (remark that Hadamard surfaces with finite total curvature have been employed as reference ones in [GW], $\mathrm{A}$, and so on). However, we have obtained some results as the relationships between radial curvature geometry and pure sectional curvature geometry in the first author and Ohta' $\mathrm{KO}$, the first author's [K], and Shiohama and the second author' [ST]. In particular, we will emphasize that the Cohn-Vossen's classical theorem ([CV2, Satz 5]) has been effectively applied in the proof of [KO, Theorem $\mathrm{C}]$.

On the other hand, many theorems, except for the Gauss-Bonnet Theorem, in geometry of surfaces are generalized, by the same way in proof, on pure sectional curvature geometry in higher dimensions. However, some results peculiar to geometry of surfaces have not been generalized even on pure sectional curvature geometry in higher ones (such results can be found in [SST]). One may say that you are so greedy, and yet we want to know that what one can bridge the relationship between radial curvature geometry, pure sectional curvature geometry, and geometry of surfaces with. In this article, we bridge the relationship between those geometries with the structure of the cut locus and the total curvature of a von Mangoldt surface of revolution, and also GTCT-II.

The Gauss - Bonnet Theorem says that the total curvature of a compact Riemannian 2-manifold $S$ is a topological invariant, that is, equal to $2 \pi \chi(S)$. Here, $\chi(S)$ is the Euler characteristic of $S$. For a complete open (i.e., non-compact) Riemannian 2-manifold, however, with admitting a total curvature, the total curvature of the surface is not a topological invariant anymore. Cohn-Vossen proved that if a complete open, finitelyconnected Riemannian 2-manifold $X$ admits a total curvature, then the total curvature of $X$ is not greater than $2 \pi \chi(X)$ (see [CV1, Satz 6]). He has developed fundamental techniques for investigating the structures of complete open Riemannian 2-manifolds. Although he restricted himself to 2-dimensional Riemannian manifolds, some techniques are ever now useful for investigating the relationship between the topology and the sectional curvature of any-dimensional complete open Riemannian manifolds. As pointed out in the preface of [SST], it took more than thirty years to obtain higher-dimensional extensions of Cohn-Vossen's results. They are the Toponogov splitting theorem [To] and the structure theorem for complete open Riemannian $n$-manifolds of positive sectional curvature GM and of non-negative sectional curvature [CG2]. As pointed out above, $2 \pi \chi(X)-c(X)$, where $c(X)$ denotes the total curvature of a complete open, finitely-connected Riemannian 2 -manifold $X$, is not a topological invariant, but it depends only on the ends of $X$. This is a direct consequence from the isoperimetric inequalities (see [SST, Theorem 5.2.1]).

Our main purpose of this article, from radial curvature geometry's standpoint, is to generalize the following Shiohama's result in geometry of complete open surfaces to anydimensional complete open Riemannian manifolds :

Theorem 1.1 ([S1, Main Theorem]) Let $X$ be a connected, complete open, finitelyconnected and oriented Riemannian 2-manifold with one end. If the total curvature of $X$ is greater than $(2 \chi(X)-1) \pi$, then all Busemann functions on $X$ are exhaustions. In particular, if the total curvature of $X$ is greater than $\pi$, then $X$ is homeomorphic to $\mathbb{R}^{2}$ and also all Busemann functions are exhaustions. Here, a function $F: X \longrightarrow \mathbb{R}$ is called an exhaustion, if $F^{-1}(-\infty, a]$ is compact for all $a \in \mathbb{R}$. 
In Section 3 of this article, one can find the definition and some properties of a Busemann function on an arbitrary complete open Riemannian $n$-manifold. Remark that it is also obtained in [S1, Main Theorem] that all Busemann functions on $X$ are non-exhaustions, if the total curvature of $X$ is less than $(2 \chi(X)-1) \pi$.

We call a complete open Riemannian 2-manifold $(\widetilde{M}, \tilde{p})$ with a base point $\tilde{p} \in \widetilde{M}$ a non-compact von Mangoldt surface of revolution if its Riemannian metric $d \tilde{s}^{2}$ is expressed in terms of the geodesic polar coordinates around $\tilde{p}$ as

$$
d \tilde{s}^{2}=d t^{2}+f(t)^{2} d \theta^{2}, \quad(t, \theta) \in(0, \infty) \times \mathbb{S}_{\tilde{p}}^{1}
$$

and if its Gaussian curvature

$$
G(\tilde{\gamma}(t))=-\frac{f^{\prime \prime}(t)}{f(t)}
$$

is monotone non-increasing along each meridian $\tilde{\gamma}:(0, \infty) \longrightarrow \widetilde{M}$ emanating from $\tilde{p}=$ $\tilde{\gamma}(0)$. Here, $f:(0, \infty) \longrightarrow \mathbb{R}$ is a positive smooth function and $\mathbb{S}_{\tilde{p}}^{1}:=\left\{v \in T_{\tilde{p}} \widetilde{M} \mid\|v\|=1\right\}$. In particular, $G \circ \tilde{\gamma}:[0, \infty) \longrightarrow \mathbb{R}$ is called the radial curvature function of $(\widetilde{M}, \tilde{p})$. Remark that $f$ satisfies the Jacobi equation $f^{\prime \prime}(t)+G(\tilde{\gamma}(t)) f(t)=0$ with $f(0)=0$ and $f^{\prime}(0)=1$. A paraboloid and a 2-sheeted hyperboloid are typical examples of $(\widetilde{M}, \tilde{p})$. The following untypical example of $(\widetilde{M}, \tilde{p})$ is due to Robert Sinclair :

Example 1.2 (Sinclair) We define $f(t):=e^{-t^{2}} \tanh t$ on $[0, \infty)$. It is clear that $f$ satisfies $f(0)=0$ and $\lim _{t \rightarrow \infty} f(t)=0$. Moreover, we have

$$
\begin{aligned}
& f^{\prime}(t)=\frac{1}{e^{t^{2}}}\left(-2 t \tanh t+\frac{1}{\cosh ^{2} t}\right) \\
& f^{\prime \prime}(t)=\left(4 t^{2}-2-\frac{2}{\cosh ^{2} t}\right) f(t)-\frac{4 t}{e^{t^{2}} \cosh ^{2} t} .
\end{aligned}
$$

Thus, by (1.1), we see $f^{\prime}(0)=1$ and $\lim _{t \rightarrow \infty} f^{\prime}(t)=0$. Furthermore, by (1.2), we get

$$
G(t):=-\frac{f^{\prime \prime}(t)}{f(t)}=\frac{8 t}{\sinh 2 t}+\frac{2}{\cosh ^{2} t}-4 t^{2}+2
$$

Then, we see

$$
\frac{d}{d t} G(t)=\frac{8 \sinh 2 t-16 t \cosh 2 t}{\sinh ^{2} 2 t}-\frac{2 \sinh 2 t}{\cosh ^{2} t}-8 t<0
$$

on $(0, \infty)$. Thus, $G$ is strictly monotone decreasing on $(0, \infty)$, and satisfies $\lim _{t \downarrow 0} G(t)=8$ and $\lim _{t \rightarrow \infty} G(t)=-\infty$. Therefore, a complete open Riemannian 2-manifold $(\widetilde{M}, \tilde{p})$ with a base point $\tilde{p}$ and $d \tilde{s}^{2}=d t^{2}+f(t)^{2} d \theta^{2},(t, \theta) \in(0, \infty) \times \mathbb{S}_{\tilde{p}}^{1}$, is a non-compact von Mangoldt surface of revolution, and its radial curvature function $G$ changes signs on $[0, \infty)$. In particular, since $f$ satisfies $\lim _{t \rightarrow \infty} f^{\prime}(t)=0$, the total curvature of this $(\widetilde{M}, \tilde{p})$ is equal to $2 \pi$ (Indeed, one can show this by calculating the total curvature, or by the isoperimetric inequality in [SST, (5.2.2) of Theorem 5.2.1]). 
Other examples of $(\widetilde{M}, \tilde{p})$ are found in the second author's [T].

Let $(M, p)$ be a complete open Riemannian $n$-manifold with a base point $p \in M$. We say that $(M, p)$ has radial (sectional) curvature at the base point $p$ bounded from below by $G \circ \tilde{\gamma}:[0, \infty) \longrightarrow \mathbb{R}$ of $(\widetilde{M}, \tilde{p})$ if, along every unit speed minimal geodesic $\gamma:[0, a) \longrightarrow M$ emanating from $p=\gamma(0)$, its sectional curvature $K_{M}$ satisfies

$$
K_{M}\left(\gamma^{\prime}(t), Y\right) \geq G(\tilde{\gamma}(t))
$$

for all $t \in[0, a)$ and $Y \in T_{\gamma(t)} M$ with $Y \perp \gamma^{\prime}(t)$. Here, $0<a \leq \infty$ is constant.

The second author [T] has investigated the structure of the cut locus of $(\widetilde{M}, \tilde{p})$. By the structure, we see that the cut locus of each point on $\widetilde{M} \backslash\{\tilde{p}\}$ is connected so that the set of all unit vectors tangent to the rays emanating from a point $\tilde{q} \in \widetilde{M}$ is too. Then, if the total curvature of $(\widetilde{M}, \tilde{p})$ is greater than $\pi$, we can bridge the relationship between radial curvature geometry and a certain result on the mass of rays in geometry of surfaces with GTCT-II, and our main theorem is now stated as following:

\section{Main Theorem.}

Let $(M, p)$ be a complete open Riemannian n-manifold $M$ whose radial curvature at the base point $p$ is bounded from below by that of a non-compact von Mangoldt surface of revolution $(\widetilde{M}, \tilde{p})$. If the total curvature of $(\widetilde{M}, \tilde{p})$ is greater than $\pi$,

(MT-1) All Busemann functions on $M$ are exhaustions.

(MT-2) There exists a compact subset $C$ of $M$ such that $C$ contains all critical points for any Busemann function on $M$.

Needless to say, the (MT-1) not only generalizes Theorem 1.1 to higher dimensions, but also is a new result in Global Riemannian Geometry of higher dimensions. A related result for the (MT-1) is Shioya's [Sya. Here, he investigated the relationship between the mass of rays and the ideal boundary of complete open Riemannian $n$-manifold with non-negative sectional curvature everywhere. The (MT-2) has never been proved even on geometry of surfaces, therefore, the (MT-2) is a purely new result in Global Riemannian Geometry. Remark that, under the assumptions in Main Theorem, $M$ has just one end by the first author's $[\mathrm{KO},(\mathrm{C}-\mathrm{i})$ in Theorem $\mathrm{C}]$.

Furthermore, it follows from Main Theorem that we have the following corollary:

\section{Corollary to Main Theorem.}

Let $(M, p)$ be a complete open Riemannian $n$-manifold $M$ whose radial curvature at the base point $p$ is bounded from below by that of a non-compact von Mangoldt surface of revolution $(\widetilde{M}, \tilde{p})$. If the total curvature of $(\widetilde{M}, \tilde{p})$ is greater than $\pi$,

(C-1) $M$ has finite topological type, that is, $M$ is homeomorphic to the interior of a compact manifold with boundary.

(C-2) The isometry group of $M$ is compact. 
One of related results for the $(\mathrm{C}-1)$, but for a complete open Riemannian $n$-manifold with non-negative sectional curvature everywhere, has been obtained in Gromov's Gv, Subsection 1.5]. Thus, the $(\mathrm{C}-1)$ is, in a sense, a weak version of Cheeger and Gromoll' [CG2, Soul Theorem] for a new class of metrics, for the radial curvature can change signs (see Example 1.2). Another related result for the $(\mathrm{C}-1)$ is Abresch and Gromoll' [AG for a complete open Riemannian $n$-manifold with non-negative Ricci curvature, sectional curvature bounded from below by some constant, and diameter growth of order $o\left(r^{1 / n}\right)$ (Their results also extends to asymptotically non-negative Ricci curvature sort). Although our radial curvature sort is 'sectional', we will emphasize again that our radial curvature can change signs (see Example 1.2). One of related results for the $(\mathrm{C}-2)$ in Main Theorem, but for a complete open Riemannian $n$-manifold with non-negative sectional curvature everywhere, has been obtained in [CG2, Corollary 6.2]. Another related result for the $(\mathrm{C}-2)$ is Yamaguchi's [Y, Theorem B], where he has proved that if a complete open Riemannian $n$-manifold admits a strictly convex function with compact levels and no minimum, then the isometry group of such a manifold is compact.

Attention to Our Results: By the remark right after Theorem 1.1, if the total curvature of $(\widetilde{M}, \tilde{p})$ is less than $\pi$, then the $(\mathrm{MT}-1)$ does not hold. Furthermore, if the total curvature of $(\widetilde{M}, \tilde{p})$ is equal to $\pi$, then the (MT-1) does not always hold. Such examples of complete open surfaces have been constructed by N. H. Kuiper and Shiohama (cf. [S1, Section 6]). Thus, one may ask us whether the $(\mathrm{C}-1)$ or the $(\mathrm{C}-2)$ still holds for a complete open Riemannian $n$-manifold $(V, p)$ whose radial curvature at the base point $p$ is bounded from below by that of a non-compact von Mangoldt surface of revolution $(\widetilde{V}, \tilde{p})$, if the total curvature of $(\widetilde{V}, \tilde{p})$ is finite. This problem will be discussed in the authors' article [KT], where our comparison model surfaces have a wider class of metrics than that of ones of non-compact von Mangoldt surfaces of revolution, and also of Cartan-Hadamard surfaces of revolution. One who minds this problem will refer to [KT].

The organization of this article is as follows. In Section 2, we give preliminaries, which are the second author's structure theorem of the cut locus of $(\widetilde{M}, \tilde{p})$ (Theorem 2.1) , GTCTII, the Rademacher theorem (Lemma 2.5) for a Lipschitz function, and a necessary and sufficient condition (Lemma 2.6) for a Lipschitz function to be differentiable at a point. In Section 3, by Lemma 2.6, we show a necessary and sufficient condition (Theorem 3.1) for a Busemann function on an arbitrary complete open Riemannian manifold to be differentiable at a point. Remark that many fundamental properties of a Busemann function had been investigated in [B] and [CG1] (see also [S2]), but, Theorem 3.1, which is one of very important tools in this article, has never been proved in general. Section 4 has two subsections. In Subsection 4.1, we first bridge the relationship (Lemma 4.1 and 4.3) between the mass of rays of $(\widetilde{M}, \tilde{p})$ and the result ([SST, Theorem 6.2.1]) on the mass of rays in the geometry of surfaces with Theorem 2.1 and the total curvature assumption of $(\widetilde{M}, \tilde{p})$, and then prove the (MT-2) (Lemma 4.4 and Proposition 4.5) in Main Theorem by GTCT-II and Lemma 4.3. Next, we prove the (MT-1) in Main Theorem by Lemma 4.4, Theorem 3.1, and lemmas, given in Section 2, for a Lipschitz function. In Subsection 4.2, we prove Corollary to Main Theorem by the (MT-1) and the (MT-2), that is, the $(\mathrm{C}-1)$ is gotten by Lemma 4.4 and $[\mathrm{Gv}$, Isotopy Lemma], and the $(\mathrm{C}-2)$ is gotten by the 
(MT-1), Myers and Steenrod' method in [MS, Section 7], and Proposition 4.5.

In the following sections, all geodesics will be normalized, unless otherwise stated.

Acknowledgement. We would like to thank Professor Robert Sinclair for his excellent untypical example of a non-compact von Mangoldt surface of revolution. We strongly believe that our results will have a great appeal to readers by his example.

\section{Preliminaries}

One of the basic tools used in this article is the Alexandrov-Toponogov comparison theorem for geodesic triangles of the form $\triangle(p x y) \subset M$ whose reference surface is a von Mangoldt surface of revolution. Elerath E] first discusses such a type of the Toponogov comparison theorem for a von Mangoldt surface of revolution in $\mathbb{R}^{3}$ which is a flat cone with a convex cap near the vertex. The second author proves that

Theorem 2.1 ([T, Main Theorem]) The cut locus $\operatorname{Cut}(\tilde{z})$ to each point $\tilde{z} \in \widetilde{M} \backslash\{\tilde{p}\}$ of a non-compact von Mangoldt surface of revolution $(\widetilde{M}, \tilde{p})$ is either an empty set, or a ray properly contained in the meridian $\theta^{-1}(\theta(\tilde{z})+\pi)$ laying opposite to $\tilde{z}$, and that the end point of $\operatorname{Cut}(\tilde{z})$ is the first conjugate point to $\tilde{z}$ along the unique minimal geodesic from $\tilde{z}$ sitting in $\theta^{-1}(\theta(\tilde{z})) \cup \theta^{-1}(\theta(\tilde{z})+\pi)$.

This special property makes it possible not only to bridge radial curvature geometry and the result ([SST, Theorem 6.2.1]) on the mass of rays in geometry of surfaces (see Lemma 4.1, 4.3, and 4.4 in Subsection 4.1), but also to find a geodesic triangle $\widetilde{\triangle}(p x y):=$ $\triangle(\tilde{p} \tilde{x} \tilde{y}) \subset \widetilde{M}$ corresponding to an arbitrarily given geodesic triangle $\triangle(p x y) \subset M$.

Theorem 2.2 (GTCT-II, [IMS, Theorem 1.3]) Let $(M, p)$ be a complete open Riemannian $n$-manifold whose radial curvature at $p$ is bounded from below by that of a noncompact von Mangoldt surface of revolution $(\widetilde{M}, \tilde{p})$. Then, for every geodesic triangle $\triangle($ pxy $) \subset M$, there exists a geodesic triangle $\widetilde{\triangle}(p x y)=\triangle(\tilde{p} \tilde{x} \tilde{y}) \subset \widetilde{M}$ such that

$$
d(\tilde{p}, \tilde{x})=d(p, x), \quad d(\tilde{p}, \tilde{y})=d(p, y), \quad d(\tilde{x}, \tilde{y})=d(x, y)
$$

and that

$$
\angle(p x y) \geq \angle(\tilde{p} \tilde{x} \tilde{y}), \quad \angle(p y x) \geq \angle(\tilde{p} \tilde{y} \tilde{x}), \quad \angle(x p y) \geq \angle(\tilde{x} \tilde{p} \tilde{y}) .
$$

Here, we denote by $d$ the distance function induced from the Riemannian structure of $M$, and also by $\angle(p x y)$ the angle between the geodesics from $x$ to $p$ and $y$ forming the triangle $\triangle(p x y)$.

Remark 2.3 GTCT-II for a compact case has been applied in the first author and Ohta' [KO]. In particular, the Grove-Shiohama Diameter Sphere Theorem [GS] has been generalized to a wide class of metrics (see [KO, Theorem A]). Furthermore, Sinclair and the second author [SiT] have obtained the structure theorem of the cut locus of a 2-sphere of revolution, and also proved the Alexandrov-Toponogov comparison theorem for a compact Riemannian $n$-manifold whose radial curvature at a base point of the manifold is 
bounded from below by that of a 2-sphere of revolution. Their comparison theorem has been applied in the first author's $[\mathrm{K}]$ to show sphere theorems, which have a different class of metrics from the one described in [KO].

The following lemmas on analysis will be applied in Section 3 to show the differentiability of a Busemann function of a ray at a starting point of some asymptotic ray of the ray (Theorem 3.1), and also in Section 4 to show our Main Theorem.

Lemma 2.4 Let $E \subset \mathbb{R}^{n}$ be a subset of Lebesgue measure zero. Then for almost all $x \in \mathbb{R}^{n-1}$, the set

$$
E_{x}:=\{t \in \mathbb{R} \mid(t, x) \in E\}
$$

is a subset of $\mathbb{R}$ of Lebesgue measure zero.

The proof of Lemma 2.4 is written in [WZ, Lemma 6.5].

Lemma 2.5 (Rademacher) If a function $F: U \longrightarrow \mathbb{R}$ is Lipschitz on an open subset $U$ of $\mathbb{R}^{n}$, then $F$ is differentiable almost everywhere.

One may find the proof of this Rademacher theorem in [Mo]. Furthermore, applying the second author' method in [MT, Section 5] with Matsuyama, we have the following lemma, which is applied in the proof of Theorem 3.1.

Lemma 2.6 Let $F: U \longrightarrow \mathbb{R}$ be a Lipschitz function defined on an open subset $U$ of $\mathbb{R}^{n}$. Then, a necessary and sufficient condition for $F$ to be differentiable at $x_{0} \in U$ is that the directional derivative function

$$
F_{+}^{\prime}\left(x_{0} ; v\right):=\lim _{t \downarrow 0} \frac{F\left(x_{0}+t v\right)-F\left(x_{0}\right)}{t}
$$

exists for all $v \in \mathbb{R}^{n}$ and is linear.

Proof. It is sufficient to prove that $F$ is differentiable at $x_{0} \in U$, if the directional derivative function $F_{+}^{\prime}\left(x_{0} ; v\right)$ of $F$ exists for all $v \in \mathbb{R}^{n}$ and is linear. Under this assumption, we have

$$
\lim _{t \downarrow 0} \frac{\left|F\left(x_{0}+t v\right)-F\left(x_{0}\right)-F_{+}^{\prime}\left(x_{0} ; t v\right)\right|}{t}=0
$$

for all unit vectors $v \in U$. Take any fixed $\varepsilon>0$. Since $\mathbb{S}_{x_{0}}^{n-1}:=\left\{v \in T_{x_{0}}\left(\mathbb{R}^{n}\right) \mid\|v\|=1\right\}$ is compact, we can take finitely many $v_{1}, \ldots, v_{k(\varepsilon)} \in \mathbb{S}_{x_{0}}^{n-1}$ such that for any $v \in \mathbb{S}_{x_{0}}^{n-1}$, $\left\|v_{i}-v\right\|<\varepsilon$ holds for some $i \in\{1, \ldots, k(\varepsilon)\}$. Let $w$ be any nonzero vector at $x_{0}$, and $v_{i_{0}}$ be one of the vectors $v_{1}, \ldots, v_{k(\varepsilon)}$ such that

$$
\left\|\frac{1}{\|w\|} w-v_{i_{0}}\right\|<\varepsilon
$$

By the triangle inequality, we have

$$
\begin{aligned}
& \left|F\left(x_{0}+w\right)-F\left(x_{0}\right)-F_{+}^{\prime}\left(x_{0} ; w\right)\right| \\
& \leq\left|F\left(x_{0}+w\right)-F\left(x_{0}+\|w\| v_{i_{0}}\right)\right|+\left|F\left(x_{0}+\|w\| v_{i_{0}}\right)-F\left(x_{0}\right)-F_{+}^{\prime}\left(x_{0} ;\|w\| v_{i_{0}}\right)\right| \\
& \quad+\left|F_{+}^{\prime}\left(x_{0} ; w\right)-F_{+}^{\prime}\left(x_{0} ;\|w\| v_{i_{0}}\right)\right| .
\end{aligned}
$$


Now, since $F$ is Lipschitz, there exists Lipschitz constant number $C_{0}$ such that

$$
\left|F\left(x_{0}+w\right)-F\left(x_{0}+\|w\| v_{i_{0}}\right)\right| \leq C_{0}\|w-\| w\left\|v_{i_{0}}\right\|<C_{0}\|w\| \varepsilon .
$$

Furthermore, since $F_{+}^{\prime}\left(x_{0} ; \cdot\right)$ is linear,

$$
\left|F_{+}^{\prime}\left(x_{0} ; w\right)-F_{+}^{\prime}\left(x_{0} ;\|w\| v_{i_{0}}\right)\right| \leq C_{0}\|w-\| w\left\|v_{i_{0}}\right\|<C_{0}\|w\| \varepsilon .
$$

By (2.2), (2.3), and (2.4), we see

$$
\begin{aligned}
& \left|F\left(x_{0}+w\right)-F\left(x_{0}\right)-F_{+}^{\prime}\left(x_{0} ; w\right)\right| \\
& <2 C_{0}\|w\| \varepsilon+\left|F\left(x_{0}+\|w\| v_{i_{0}}\right)-F\left(x_{0}\right)-F_{+}^{\prime}\left(x_{0} ;\|w\| v_{i_{0}}\right)\right|,
\end{aligned}
$$

for all nonzero vectors $w$ at $x_{0}$. Thus, by (2.1) and (2.5), we get

$$
\limsup _{\|w\| \rightarrow 0} \frac{\left|F\left(x_{0}+w\right)-F\left(x_{0}\right)-F_{+}^{\prime}\left(x_{0} ; w\right)\right|}{\|w\|} \leq 2 C_{0} \varepsilon \text {. }
$$

Since $\varepsilon$ is arbitrarily taken, this inequality (2.6) shows that $F$ is differentiable at $x_{0}$.

Cohn-Vossen CV2 would be the first geometer who applied the following lemma to differential geometry. We will see in the proof of Lemma 4.6 that this lemma is applied to a Busemann function.

Lemma 2.7 Let $F:[a, b] \longrightarrow \mathbb{R}$ be a Lipschitz function. Then its derivative function $F^{\prime}(x)$ is integrable and

$$
F(x)-F(a)=\int_{a}^{x} F^{\prime}(t) d t
$$

holds for all $x \in[a, b]$.

Lemma 2.7 is proved, for example, in [WZ, Theorem 7.29].

Remark 2.8 Lipschitz continuity is a very important property. For example, the CantorLebesgue function admits an integrable derivative function, i.e., 0, but this function does not satisfy the equation (2.7).

\section{Differentiability Theorem of Busemann Functions}

In this section, let $V$ be an arbitrary complete open Riemannian $n$-manifold without curvature assumptions. A Busemann function $F_{\gamma}: V \longrightarrow \mathbb{R}$ of a ray $\gamma$ on $V$ is defined by

$$
F_{\gamma}(x):=\lim _{t \rightarrow \infty}\{t-d(x, \gamma(t))\} .
$$

$F_{\gamma}$ was first introduced by H. Busemann ([B, Section 22]) to investigate parallels for straight lines on a straight $G$-space where every two points are joined by a unique geodesic realizing the distance. By the definition of $F_{\gamma}$, we have that $\left|F_{\gamma}(x)-F_{\gamma}(y)\right| \leq d(x, y)$ for all $x, y \in V$. Thus, $F_{\gamma}$ is Lipschitz continuous with Lipschitz constant 1 so that $F_{\gamma}$ is differentiable except for a measure zero set. Furthermore, on the differentiability of $F_{\gamma}$, we have already known the following fact in general (cf. [S2, Theorem 1.1]) : 
$F_{\gamma}$ is differentiable at $x \in V$, if $x$ is an interior point of some asymptotic ray $\sigma$ of $\gamma$.

However, the differentiability at $\sigma(0)$ of $F_{\gamma}$ has never been studied in general. Applying the second author' method in [IT, Lemma 2.1] with Itoh, we have the following theorem on the differentiability at $\sigma(0)$ of $F_{\gamma}$ :

Theorem 3.1 Let $F_{\gamma}$ be a Busemann function of a ray $\gamma$ on a complete open Riemannian $n$-manifold $V$. Then, $F_{\gamma}$ is differentiable at a point $q \in V$ if and only if there exists a unique asymptotic ray $\sigma$ of $\gamma$ emanating from $q=\sigma(0)$. Moreover, the gradient vector of $F_{\gamma}$ at a differentiable point $q$ equals the velocity vector of the unique asymptotic ray of $\gamma$.

\subsection{Proof of Theorem 3.1}

We will first recall the following fundamental property of a Busemann function:

Lemma 3.2 (cf. [S2, Theorem 1.1]) For any asymptotic ray $\sigma$ of a ray $\gamma$ in $V$,

$$
F_{\gamma} \circ \sigma(t)=t+F_{\gamma} \circ \sigma(0)
$$

holds for all $t \geq 0$.

By Lemma 3.2, it is easy to prove that if $F_{\gamma}$ is differentiable at a point $q \in V$, there exists a unique asymptotic ray $\sigma$ of $\gamma$ emanating from $q=\sigma(0)$.

Next, we will prove that $F_{\gamma}$ is differentiable at a point $q \in V$, if there exists a unique asymptotic ray $\sigma$ of $\gamma$ emanating from $q=\sigma(0)$. To see this, it is, of course, sufficient to prove the following lemma.

Lemma 3.3 For every geodesic segment $\tau(s)$ emanating from $q$ in a convex neighborhood $U_{q}$ around $q$, we have

$$
\lim _{s \rightarrow 0} \frac{F_{\gamma}(\tau(s))-F_{\gamma}(q)}{s}=\left\langle\tau^{\prime}(0), \sigma^{\prime}(0)\right\rangle=\cos \left(\angle\left(\tau^{\prime}(0), \sigma^{\prime}(0)\right)\right) .
$$

Here, $\sigma$ is the unique asymptotic ray of $\gamma$ emanating from $q=\sigma(0)$.

Proof. Take a point $r \in U_{q}$ on the unique asymptotic ray $\sigma$ of $\gamma$. We define

$$
\varphi(s):=d(r, \tau(s))
$$

for any fixed minimal geodesic segment $\tau(s)$ emanating from $q$ in $U_{q}$. It follows from the Taylor expansion of $\varphi(s)$ about $s=0$ that there exists a constant $C_{1}$ such that

$$
\begin{aligned}
\varphi(s) & =\varphi(0)+\varphi^{\prime}(0) s+\frac{1}{2 !} \varphi^{\prime \prime}(0) s^{2}+\cdots \\
& \leq \varphi(0)+\varphi^{\prime}(0) s+C_{1} s^{2} .
\end{aligned}
$$

By (3.1) and the first variation formula, we have

$$
\begin{aligned}
d(r, \tau(s))=\varphi(s) & \leq \varphi(0)+\varphi^{\prime}(0) s+C_{1} s^{2} \\
& =d(r, \tau(0))+s \cos \left(\pi-\angle\left(\tau^{\prime}(0), \sigma^{\prime}(0)\right)\right)+C_{1} s^{2} \\
& =d(r, q)-s \cos \left(\angle\left(\tau^{\prime}(0), \sigma^{\prime}(0)\right)\right)+C_{1} s^{2} .
\end{aligned}
$$


Since $F_{\gamma}(r)-F_{\gamma}(q)=d(q, r)$ by Lemma 3.2, we have, by (3.2),

$$
\begin{aligned}
F_{\gamma}(\tau(s))-F_{\gamma}(q) & =F_{\gamma}(\tau(s))-F_{\gamma}(r)+d(q, r) \\
& \geq d(q, r)-d(r, \tau(s)) \\
& \geq s \cos \left(\angle\left(\tau^{\prime}(0), \sigma^{\prime}(0)\right)\right)-C_{1} s^{2} .
\end{aligned}
$$

Thus, by this (3.3), we have

$$
\liminf _{s \rightarrow 0} \frac{F_{\gamma}(\tau(s))-F_{\gamma}(q)}{s} \geq \cos \left(\angle\left(\tau^{\prime}(0), \sigma^{\prime}(0)\right)\right) .
$$

On the other hand, for each sufficiently small $s$, let $\sigma_{s}$ be an asymptotic ray of $\gamma$ emanating from $\tau(s)$, and set $\eta_{s}:=\angle\left(\sigma_{s}^{\prime}(0), \tau^{\prime}(s)\right)$ and $\delta:=d(q, r)$. Furthermore, we define, for fixed $s$,

$$
\psi_{s}(t):=d\left(\sigma_{s}(\delta), \tau(t)\right) .
$$

Since the distance function $d(\cdot, \cdot)$ is $C^{\infty}$ around $(q, r)$, it follows from the Taylor expansion of $\psi_{s}(t)$ about $t=s$ that there exists a constant $C_{2}$ such that

$$
\begin{aligned}
\psi_{s}(t) & =\psi_{s}(s)+\psi_{s}^{\prime}(s)(t-s)+\frac{1}{2 !} \psi_{s}^{\prime \prime}(s)(t-s)^{2}+\cdots \\
& \leq \psi_{s}(s)+\psi_{s}^{\prime}(s)(t-s)+C_{2}(t-s)^{2}
\end{aligned}
$$

Substituting $t=0$ for (3.5), we have, by the first variation formula,

$$
\begin{aligned}
d\left(\sigma_{s}(\delta), q\right)=d\left(\sigma_{s}(\delta), \tau(0)\right)=\psi_{s}(0) & \leq \psi_{s}(s)-s \psi_{s}^{\prime}(s)+C_{2} s^{2} \\
& \leq d\left(\sigma_{s}(\delta), \tau(s)\right)-s \cos \left(\pi-\eta_{s}\right)+C_{2} s^{2} \\
& =d\left(\sigma_{s}(\delta), \sigma_{s}(0)\right)+s \cos \eta_{s}+C_{2} s^{2}
\end{aligned}
$$

for each sufficiently small $s$. Since $\sigma_{s}$ is an asymptotic ray of $\gamma$ emanating from $\tau(s)$, we have, by Lemma 3.2,

$$
F_{\gamma}\left(\sigma_{s}(\delta)\right)=\delta+F_{\gamma}\left(\sigma_{s}(0)\right)=\delta+F_{\gamma}(\tau(s)) .
$$

By (3.6) and (3.7), we have

$$
\begin{aligned}
F_{\gamma}(\tau(s))-F_{\gamma}(q) & =F_{\gamma}\left(\sigma_{s}(\delta)\right)-F_{\gamma}(q)-\delta \\
& \leq d\left(q, \sigma_{s}(\delta)\right)-d(q, \sigma(\delta)) \\
& =d\left(q, \sigma_{s}(\delta)\right)-d\left(\sigma_{s}(0), \sigma_{s}(\delta)\right) \\
& \leq s \cos \eta_{s}+C_{2} s^{2}
\end{aligned}
$$

for each sufficiently small $s$. Thus, since $\sigma$ is the unique asymptotic ray of $\gamma$ emanating from $q=\sigma(0)$, we have, by (3.8),

$$
\limsup _{s \rightarrow 0} \frac{F_{\gamma}(\tau(s))-F_{\gamma}(q)}{s} \leq \lim _{s \rightarrow 0} \cos \eta_{s}=\cos \left(\angle\left(\tau^{\prime}(0), \sigma^{\prime}(0)\right)\right) .
$$

By (3.4) and (3.9), we get the desired assertion. 
Finally, we will show the last assertion in Theorem 3.1. It follows from Lemma 3.3 that

$$
d F_{\gamma}(v)=\lim _{s \rightarrow 0} \frac{F_{\gamma} \circ \exp _{q}(s v)-F_{\gamma}(q)}{s}=\left\langle v, \sigma^{\prime}(0)\right\rangle
$$

for all $v \in T_{q} V$. Thus, by this (3.10) and Lemma 2.6, we have

$$
\left(\nabla F_{\gamma}\right)_{\sigma(0)}=\sigma^{\prime}(0)
$$

for the unique asymptotic ray $\sigma$ of $\gamma$.

\section{Proofs of Main Theorem and its Corollary}

In this section, let $(M, p)$ be a complete open Riemannian $n$-manifold $M$ whose radial curvature at the base point $p$ is bounded from below by that of a non-compact von Mangoldt surface of revolution $(\widetilde{M}, \tilde{p})$ with its total curvature $c(\widetilde{M})>\pi$.

\subsection{Proof of Main Theorem}

Let $A_{\tilde{q}}$ be the set of all unit vectors tangent to the rays emanating from a point $\tilde{q} \in \widetilde{M}$. Remark that $A_{\tilde{q}}$ is connected by Theorem 2.1. Furthermore, we denote by $\mu$ the Lebesgue measure on the unit circle $\mathbb{S}_{\tilde{q}}^{1}:=\left\{v \in T_{\tilde{q}} \widetilde{M} \mid\|v\|=1\right\}$ with total measure $2 \pi$. Since $c(\widetilde{M})>\pi$, it follows from [SST, Theorem 6.2.1] that

Lemma 4.1 Let $(\widetilde{M}, \tilde{p})$ be a non-compact von Mangoldt surface of revolution with its total curvature $c(\widetilde{M})>\pi$. Then, there exist numbers $R, \delta>0$ such that

$$
\mu\left(A_{\tilde{q}}\right)<\pi-2 \delta
$$

for all $\tilde{q} \in \widetilde{M} \backslash B_{R}(\tilde{p})$. Here, $B_{R}(\tilde{p}) \subset \widetilde{M}$ is the open distance $R$-ball around $\tilde{p} \in \widetilde{M}$.

Remark 4.2 Maeda [M] very first bridged the relationship between the total curvature and the mass of rays in geometry of surfaces. His result was extended in Shiga's $[\mathrm{Sg}$, where the Gaussian curvature changes signs. We will refer some relationships between the total curvature and the mass of rays in geometry of surfaces to [SST].

As a direct consequence from Lemma 4.1, we have the following lemma:

Lemma 4.3 Let $R, \delta>0$ be the numbers in Lemma 4.1. For each $\tilde{q} \in \widetilde{M} \backslash \overline{B_{R}(\tilde{p})}$, there exists a number $R_{1}>R$ such that

$$
\angle(\tilde{p} \tilde{q} \tilde{x}) \geq \frac{\pi}{2}+\delta
$$

for all $\tilde{x} \in \widetilde{M} \backslash B_{R_{1}}(\tilde{p})$. Here, $\angle(\tilde{p} \tilde{q} \tilde{x})$ is the angle between minimal geodesics from $\tilde{q}$ to $\tilde{p}$ and $\tilde{x}$.

In all of the following lemmas and propositions, let $R, \delta>0$ be also the numbers in Lemma 4.1 . 
Lemma 4.4 For any ray $\gamma$ on $M$ and any asymptotic ray $\sigma$ of $\gamma$ emanating from a point $q=\sigma(0) \in M \backslash \overline{B_{R}(p)}$, we have

$$
\angle\left(\sigma^{\prime}(0), \alpha^{\prime}(d(p, q))\right) \leq \frac{\pi}{2}-\delta
$$

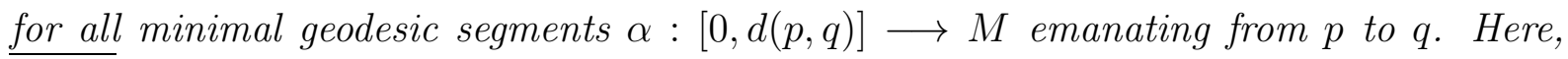
$\overline{B_{R}(p)} \subset M$ is the closed distance $R$-ball around $p \in M$.

Proof. For any sufficiently small fixed number $\varepsilon>0$, let $\alpha_{s}$ be a minimal geodesic emanating from $p$ to $\sigma(s)$ for each $s \in(0, \varepsilon)$, and let $t_{0}>0$ be a sufficiently large number such that

$$
d\left(p, \gamma\left(t_{0}\right)\right) \gg \max _{s \in[0, \varepsilon]} d(p, \sigma(s)) .
$$

Furthermore, for each $s \in(0, \varepsilon)$, let $\sigma_{s, t_{0}}$ be a minimal geodesic emanating from $\sigma(s)$ to $\gamma\left(t_{0}\right)$. Consider a geodesic triangle $\widetilde{\triangle}\left(p \sigma(s) \gamma\left(t_{0}\right)\right) \subset \widetilde{M}$ corresponding to the geodesic triangle $\triangle\left(p \sigma(s) \gamma\left(t_{0}\right)\right)$ consisting of $\alpha_{s}, \sigma_{s, t_{0}}$, and a minimal geodesic joining $p$ to $\gamma\left(t_{0}\right)$. Then, we have, by GTCT-II and Lemma 4.3,

$$
\angle\left(-\alpha_{s}^{\prime}(d(p, \sigma(s))), \sigma_{s, t_{0}}^{\prime}(0)\right) \geq \angle\left(-\tilde{\alpha}_{s}^{\prime}(d(p, \sigma(s))), \tilde{\sigma}_{s, t_{0}}^{\prime}(0)\right) \geq \frac{\pi}{2}+\delta
$$

for each $s \in(0, \varepsilon)$. Here, we denote by $\tilde{\alpha}_{s}$ and $\tilde{\sigma}_{s, t_{0}}$ the edges corresponding to $\alpha_{s}$ and $\sigma_{s, t_{0}}$, respectively. On the other hand, since, for each $s \in(0, \varepsilon)$, the sub-ray $\left.\sigma\right|_{[s, \infty)}$ is the unique asymptotic ray of $\gamma$ emanating from $\sigma(s)$ (cf. [S2, Theorem 1.1]), each $\sigma_{s, t_{0}}$ converges to the sub-ray $\left.\sigma\right|_{[s, \infty)}$ as $t_{0} \rightarrow \infty$. Since $t_{0}$ is taken sufficiently large, we see, by (4.1), for each fixed $s \in(0, \varepsilon)$,

$$
\angle\left(-\alpha_{s}^{\prime}(d(p, \sigma(s))), \sigma^{\prime}(s)\right) \geq \frac{\pi}{2}+\delta
$$

Moreover, since $\mathbb{S}_{p}^{n-1}:=\left\{v \in T_{p} M \mid\|v\|=1\right\}$ is compact, there exists a sequence $\left\{s_{n}\right\} \rightarrow$ 0 as $n \rightarrow \infty$ such that $\alpha_{s_{n}}$ converges to some minimal geodesic $\alpha_{0}$ emanating $p$ to $q$. Then, since $\sigma^{\prime}\left(s_{n}\right)$ converges to $\sigma^{\prime}(0)$ as $n \rightarrow \infty$, we have, by (4.2),

$$
\angle\left(-\alpha_{0}^{\prime}(d(p, q)), \sigma^{\prime}(0)\right) \geq \frac{\pi}{2}+\delta .
$$

Thus, by (4.3) and the second author's [IT, Lemma 2.1], we have, for any minimal geodesic segment $\alpha:[0, d(p, q)] \longrightarrow M$ emanating from $p$ to $q$,

$$
\angle\left(-\alpha^{\prime}(d(p, q)), \sigma^{\prime}(0)\right) \geq \angle\left(-\alpha_{0}^{\prime}(d(p, q)), \sigma^{\prime}(0)\right) \geq \frac{\pi}{2}+\delta .
$$

Therefore, we get, by this (4.4),

$$
\angle\left(\sigma^{\prime}(0), \alpha^{\prime}(d(p, q))\right)=\pi-\angle\left(-\alpha^{\prime}(d(p, q)), \sigma^{\prime}(0)\right) \leq \frac{\pi}{2}-\delta
$$

which is the desired assertion in this lemma. 
Recall that a point $x \in M$ is called a critical point for a Busemann function $F_{\gamma}$ of a ray $\gamma$ on $M$ if, for every nonzero unit vector $v \in T_{x} M$, there exists an asymptotic ray $\sigma$ of $\gamma$ emanating from $\sigma(0)=x$ such that $\angle\left(v, \sigma^{\prime}(0)\right) \leq \pi / 2$ (cf. [S3]). Therefore, as a direct consequence from Lemma 4.4, we have the following proposition, which shows the (MT-2) in Main Theorem:

Proposition 4.5 $\overline{B_{R}(p)}$ contains all critical points for any Busemann function on $M$.

In the following, we are going to show the (MT-1) in Main Theorem.

Lemma 4.6 Let $F_{\gamma}$ be a Busemann function of a ray $\gamma$ on $M$ and $\alpha(t)$ a minimal geodesic segment in $M$ emanating from $p$. If $F_{\gamma}$ is differentiable at $\alpha(t)$ for almost all $t \in(a, b)$, and satisfies

$$
\angle\left(\left(\nabla F_{\gamma}\right)_{\alpha(t)}, \alpha^{\prime}(t)\right) \leq \frac{\pi}{2}-\delta
$$

then, we have

$$
F_{\gamma}(\alpha(b))-F_{\gamma}(\alpha(a)) \geq(b-a) \sin \delta
$$

Proof. By assumptions on this lemma and Theorem 3.1, we have

$$
\begin{aligned}
\frac{d}{d t} F_{\gamma}(\alpha(t)) & =\left\langle\left(\nabla F_{\gamma}\right)_{\alpha(t)}, \alpha^{\prime}(t)\right\rangle \\
& =\cos \left(\angle\left(\left(\nabla F_{\gamma}\right)_{\alpha(t)}, \alpha^{\prime}(t)\right)\right) \\
& \geq \cos \left(\frac{\pi}{2}-\delta\right) \\
& =\sin \delta
\end{aligned}
$$

for almost all $t \in(a, b)$. Therefore, by Lemma 2.7 and (4.5), we get

$$
F_{\gamma}(\alpha(b))-F_{\gamma}(\alpha(a))=\int_{a}^{b} \frac{d}{d t} F_{\gamma}(\alpha(t)) d t \geq(b-a) \sin \delta .
$$

Proposition 4.7 For any Busemann function $F_{\gamma}$ on $M$ and any $q \in M \backslash \overline{B_{R}(p)}$, we have

$$
F_{\gamma}(q)-F_{\gamma}(\alpha(R)) \geq(d(p, q)-R) \sin \delta
$$

Here $\alpha:[0, d(p, q)] \longrightarrow M$ denotes a minimal geodesic segment joining $p$ to $q$.

Proof. It is sufficient to prove that, for each $t_{0} \in(R, d(p, q))$, there exists a number $\varepsilon_{0}>0$ such that

$$
F_{\gamma}(\alpha(t))-F_{\gamma}(\alpha(s)) \geq(t-s) \sin \delta
$$

for all $t_{0}-\varepsilon_{0}<s<t<t_{0}+\varepsilon_{0}$. Since $\alpha\left(t_{0}\right)$ is not a cut point of $p=\alpha(0)$, there exist an open neighborhood $\mathcal{U}$ around $\alpha^{\prime}(0)$ in $\mathbb{S}_{p}^{n-1}$, an open neighborhood $V$ around $\alpha\left(t_{0}\right)$ in $M$ and an open interval $\left(t_{0}-\varepsilon_{0}, t_{0}+\varepsilon_{0}\right)$ such that $\mathcal{U} \times\left(t_{0}-\varepsilon_{0}, t_{0}+\varepsilon_{0}\right)$ is diffeomorphic to $V$ by a map $\varphi$, where $\varphi^{-1}(v, t):=\exp _{p}(t v)$. Since $F_{\gamma} \circ \varphi^{-1}$ is Lipschitz, it follows from Lemma 2.5 that there exists a subset $\mathcal{E}$ of $\mathbb{R}^{n}$ of Lebesgue measure zero such that 
$F_{\gamma} \circ \varphi^{-1}$ is differentiable on $\left(t_{0}-\varepsilon_{0}, t_{0}+\varepsilon_{0}\right) \times \mathcal{U} \backslash \mathcal{E}$. Here, $\mathcal{U}$ is identified as an open subset of $\mathbb{R}^{n-1}$. By Lemma 2.4, there exists a sequence $\left\{\alpha_{i}\right\}$ of minimal geodesic segments emanating from $\alpha(0)$ converging to $\alpha$ such that $F_{\gamma}$ is differentiable almost everywhere on $\alpha_{i}\left(t_{0}-\varepsilon_{0}, t_{0}+\varepsilon_{0}\right)$ for each $i$. By Lemmas 4.4 and 4.6, we have

$$
F_{\gamma}\left(\alpha_{i}(t)\right)-F_{\gamma}\left(\alpha_{i}(s)\right) \geq(t-s) \sin \delta
$$

for all $t_{0}-\varepsilon_{0}<s<t<t_{0}+\varepsilon_{0}$ and all $i$. By taking the limit, we get

$$
F_{\gamma}(\alpha(t))-F_{\gamma}(\alpha(s)) \geq(t-s) \sin \delta
$$

for all $t_{0}-\varepsilon_{0}<s<t<t_{0}+\varepsilon_{0}$.

Recall again that a function $F: M \longrightarrow \mathbb{R}$ is said to be an exhaustion if $F^{-1}(-\infty, a]$ is compact for all $a \in \mathbb{R}$. Now, we set

$$
M\left(F_{\gamma}: a\right):=\left\{x \in M \mid F_{\gamma}(x) \leq a\right\}
$$

for all $a \in \mathbb{R}$. In Proposition 4.7, since $\partial B_{R}(p)$ is compact, there exists a constant number $\mathcal{N}(R)$ such that $F_{\gamma}(\alpha(R)) \geq \mathcal{N}(R)$. By Proposition 4.7, we have

$$
a-\mathcal{N}(R) \geq F_{\gamma}(q)-F_{\gamma}(\alpha(R)) \geq(d(p, q)-R) \sin \delta
$$

for all $q \in\left(M \backslash \overline{B_{R}(p)}\right) \cap M\left(F_{\gamma}: a\right)$. By this (4.6), we get,

$$
d(p, q) \leq \frac{a-\mathcal{N}(R)}{\sin \delta}+R
$$

for all $q \in\left(M \backslash \overline{B_{R}(p)}\right) \cap M\left(F_{\gamma}: a\right)$. Thus, (4.7) implies that $M\left(F_{\gamma}: a\right)$ is compact. Therefore, all Busemann functions on $M$ are exhaustions.

\subsection{Proof of Corollary to Main Theorem}

We first show the $(\mathrm{C}-1)$. Recall that, for a fixed point $q \in M$, a point $x \in M \backslash\{q\}$ is called a critical point of $d(q, \cdot)$ (or critical point for $q$ ) if, for every nonzero tangent vector $v \in T_{x} M$, we find a minimal geodesic $\gamma$ emanating from $x$ to $q$ satisfying $\angle\left(v, \gamma^{\prime}(0)\right) \leq \pi / 2$ (see [GS] and [Gv]). Thus, Lemma 4.4 implies that there exists a number $\varepsilon>0$ such that $M \backslash B_{R+\varepsilon}(p)$ has no critical point of $d(p, \cdot)$, where $R>0$ is the number in Lemma 4.1, By [Gv, Isotopy Lemma], $M \backslash B_{R+\varepsilon}(p)$ is homeomorphic to $\partial B_{R+\varepsilon}(p) \times[R+\varepsilon, \infty)$ so that $M$ has finite topological type.

Next, we show the $(\mathrm{C}-2)$. Let $I(M)$ be the isometry group of $M(\operatorname{dim} M=n)$, that is, a Lie transformation group. We will first recall Myers and Steenrod' method in [MS, Section 7]. They took $n+1$ points $x_{0}, x_{1}, \ldots, x_{n}$ in $M$ which are independent in a certain sense (roughly speaking, $x_{1}, x_{2}, \ldots, x_{n}$ are different points to each other in a convex neighborhood around $x_{0}$ with $x_{i} \neq x_{0}$ for $i=1,2, \ldots, n$ such that each vector $v_{i}$ at $x_{0}$, which is tangent to each minimal geodesic emanating from $x_{0}$ to $x_{i}$ for $i=1,2, \ldots, n$, is linearly independent), and proved that the mapping

$$
g \in I(M) \longrightarrow\left(g\left(x_{0}\right), g\left(x_{1}\right), \ldots, g\left(x_{n}\right)\right) \in M^{n+1}
$$


is one-to- one and has a closed submanifold of $M^{n+1}$ as its image. Here, $M^{n+1}$ is the product manifold $M \times M \times \cdots \times M$ taken with itself $n+1$ times. Thus, it is sufficient to prove that $d(p, g(q))$ is bounded from above by some constant for all $g \in I(M)$ and for some point $q \in M$ with a certain special property. Now, let $F_{\gamma}$ be a Busemann function of any ray $\gamma$ on $M$. Since $F_{\gamma}$ is an exhaustion by the $(\mathrm{MT}-1), F_{\gamma}(\cdot)$ attains its minimum at some $q_{0} \in M$. Thus, $q_{0}$ is a critical point for $F_{\gamma}$, and hence $q_{0} \in \overline{B_{R}(p)}$ by Proposition 4.5, where $R>0$ is the number in Lemma 4.1. For any $g \in I(M)$, we see $g\left(q_{0}\right) \in \overline{B_{R}(p)}$, for $g\left(q_{0}\right)$ is a critical point for $F_{g \circ \gamma}$ of the ray $g \circ \gamma$ on $M$. Thus, we get

$$
d\left(p, g\left(q_{0}\right)\right) \leq R
$$

for all $g \in I(M)$. Therefore, $I(M)$ is compact.

\section{References}

[A] U. Abresch, Lower curvature bounds, Toponogov's theorem, and bounded topology, Ann. Sci. École Norm. Sup. (4) 18 (1985), 651-670.

[AG] U. Abresch and D. Gromoll, On complete manifolds with nonnegative Ricci curvature, J. Amer. Math. Soc. 3 (1990), no. 2, 355-374.

[B] H. Busemann, The Geometry of Geodesics, Pure and Appl. Math. 6, Academic Press, New York, 1955.

[CG1] J. Cheeger and D. Gromoll, The splitting theorem for manifolds of nonnegative Ricci curvature, J. Differential Geom. 6 (1971), 119-128.

[CG2] J. Cheeger and D. Gromoll, On the structure of complete manifolds of nonnegative curvature, Ann. of Math. 96 (1972), 415-443.

[CV1] S. Cohn-Vossen, Kürzeste Wege und Totalkrümmung auf Flächen, Compositio Math. 2 (1935), 63-113.

[CV2] S. Cohn-Vossen, Totalkrümmung und geodätische Linien auf einfach zusammenhängenden offenen volständigen Flächenstücken, Recueil Math. Moscow 43 (1936), 139-163.

[E] D. Elerath, An improved Toponogov comparison theorem for nonnegatively curved manifolds, J. Differential Geom. 15 (1980), 187-216.

[GW] R. E. Greene and H. Wu, Function theory on manifolds which possess a pole, Lecture Notes in Mathematics 699, Springer, Berlin, 1979.

[GM] D. Gromoll and W. Meyer, On complete manifolds of positive curvature, Ann. of Math. (2) 75 (1969), 75-90.

[Gv] M. Gromov, Curvature, diameter and Betti numbers, Comment. Math. Helv. 56 (1981), 179-195. 
[GS] K. Grove and K. Shiohama, A generalized sphere theorem, Ann. of Math. (2) 106 (1977), 201-211.

[IT] J. Itoh and M. Tanaka, The Lipschitz continuity of the distance function to the cut locus, Trans. Amer. Math. Soc. 353 (2001), 21-40.

[IMS] Y. Itokawa, Y. Machigashira, and K. Shiohama, Generalized Toponogov's theorem for manifolds with radial curvature bounded below, Explorations in complex and Riemannian geometry, 121-130, Contemp. Math. 332, Amer. Math. Soc., Providence, RI, 2003.

[KK] N. N. Katz and K. Kondo, Generalized space forms, Trans. Amer. Math. Soc. 354 (2002), 2279-2284

[K] K. Kondo, Radius sphere theorems for compact manifolds with radial curvature bounded below, Tokyo Journal of Mathematics, 30, No. 2. (2007), 465-475.

[KO] K. Kondo and S. Ohta, Topology of complete manifolds with radial curvature bounded below, GAFA (Geom. Func. Anal.) 17 (2007), 1237-1247.

[KT] K. Kondo and M. Tanaka, Total curvatures of model surfaces control topology of complete open manifolds with radial curvature bounded below. II, Preprint, 2007.

[M] M. Maeda, A geometric significance of total curvature on complete open surfaces, in Geometry of Geodesics and Related Topics (Tokyo, 1982), Advanced Studies in Pure Math., vol. 3, North-Holland, Amsterdam, 1984, pp. 451-458.

[MT] T. Matsuyama and M. Tanaka, Scattering states for the nonlinear wave equation with small data, Advances in Diff. Eq. 9 (2004), 721-744.

[Mo] F. Morgan, Geometric Measure Theory, A Beginer's Guide, Academic Press, 1988.

[MS] S. B. Myers and N. E. Steenrod, The groups of isometries of a Riemannian manifold, Ann. of Math. 40 (1939), 400-416.

[R] R. T. Rockafellar, Convex Analysis, Princeton Univ. Press, 1972.

[Sg] K. Shiga, On a relation between the total curvature and the measure of rays, Tsukuba J. Math. 6 (1982), no. 1, 41-50.

[S1] K. Shiohama, The role of total curvature on complete noncompact Riemannian 2-manifolds, Illinois J. Math. 28 (1984), 597-620.

[S2] K. Shiohama, Topology of complete noncompact manifolds, in Geometry of Geodesics and Related Topics (Tokyo, 1982), Advanced Studies in Pure Math., vol. 3, North-Holland, Amsterdam, 1984, pp. 423-450.

[S3] K. Shiohama, An integral formula for the measure of rays on complete open surfaces, J. Differential Geom. 23 (1986), 197-205. 
[SST] K. Shiohama, T. Shioya, and M. Tanaka, The Geometry of Total Curvature on Complete Open Surfaces, Cambridge University Press, Cambridge, 2003.

[ST] K. Shiohama and M. Tanaka, Compactification and maximal diameter theorem for noncompact manifolds with radial curvature bounded below, Math. Zeitschrift 241 (2002), 341-351.

[Sya] T. Shioya, On asymptotic behavior of the mass of rays, Proc. Amer. Math. Soc. 28 (1984), 597-620.

[SiT] R. Sinclair and M. Tanaka, The cut locus of a 2-sphere of revolution and Toponogov's comparison theorem, Preprint, 2006.

[T] M. Tanaka, On the cut loci of a von Mangoldt's surface of revolution, J. Math. Soc. Japan 44 (1992), 631-641.

[To] V. A. Toponogov, Riemannian spaces containing straight lines, Dokl. Akad. Nauk SSSR 127 (1959), 977-979.

[WZ] R. L. Wheeden and A. Zygmund, Measure and Integral, Marcel Decker, New York, 1977.

[Y] T. Yamaguchi, The isometry groups of Riemannian manifolds admitting strictly convex functions, Ann. Sci. École Norm. Sup. (4) 15 (1982), 205-212.

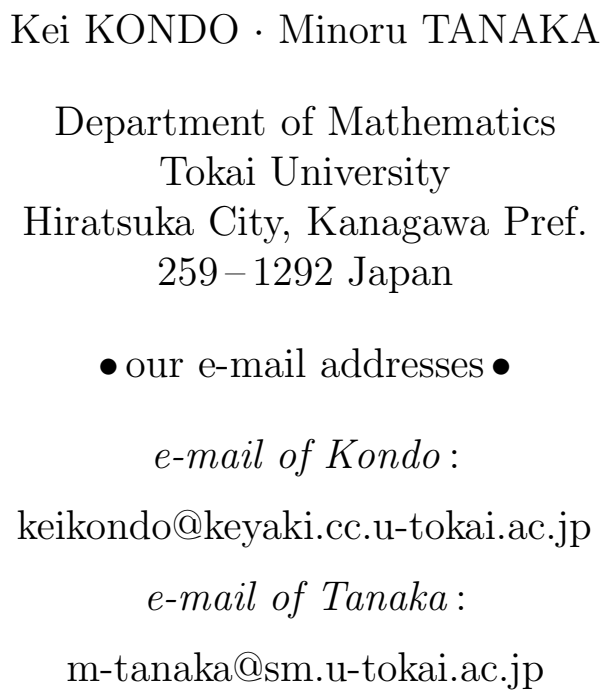

